\title{
Calidad microbiológica y físico-química del agua para usos recreativos en las playas de Bocagrande y Marbella, en Cartagena de Indias, Colombia
}

Adriana Yepes Mayorga*

adyepes@yahoo.com

\section{RESUMEN}

En la actualidad Cartagena es el puerto colombiano sobre el mar Caribe con mayor reconocimiento por su trayectoria histórica y por su actividad turística. Debido a la importancia que tiene la calidad de las aguas de recreo para los bañistas y usuarios en general, se evaluaron parámetros microbiológicos, físicos y químicos en las playas de Bocagrande y Marbella, durante los meses de febrero a abril de 2004, que corresponden a la época de verano de la región.

Se observó que los parámetros físicos y químicos tuvieron un comportamiento estacionario durante el periodo de muestreo y cumplen con los estándares dispuestos por el Decreto 1594 de 1984. En los parámetros microbiológicos, el promedio estimado de coliformes totales superó el índice que establece esta norma. Los resultados obtenidos para los parámetros E. coliy enterococos, se compararon con estudios asocia-

Licenciada en Biología. MSc en Ingeniería Ambiental de la Universidad Nacional de Colombia. Docente de la Facultad de Medio Ambiente y Recursos Naturales de la Universidad Distrital Francisco José de Caldas. 
dos a la aparición de enfermedades, realizados por Cheung (1989) y por Bandanarayake (1995), respectivamente.

\title{
Palabras clave:
}

Cartagena; aguas de uso recreativo; calidad sanitaria

\begin{abstract}
Cartagena is the more important Colombian port on the Caribbean Sea because its historical trajectory and tourist activity. Due to the importance that has the quality of the recreational waters for the swimmers, users in general, in this study were evaluated microbiology, physical, and chemical parameters during the months of February to April of 2004, that correspond to the summer time of the area.

It was observed that the physical and chemical parameters, normalizes by the Ordinance 1594 of 1984, have a stationary behavior during the period of sampling and they fulfill the standards of this norm. About the microbiology parameters, dear average of total coliforms overcame the values that establish the norm. The results obtained for the parameter $E$. coli and enterococci, it was proceeded to compare them with some studies carried out by Cheung (1989) and for Bandanarayake (1995), respectively.
\end{abstract}

\section{Key words:}

Cartagena; recretional waters; sanitary quality

\section{INTRODUCCIÓN}

Cartagena de Indias es la ciudad del Caribe colombiano más frecuentada por el atractivo que tiene su historia, su arquitectura civil y militar, así como sus playas. Estas son referencias obligadas de encuentro entre su población residente y la flotante. (ACUACAR, 1996; ALCALDÍA DE CARTAGENA, 2001)

Un número creciente de personas se desplaza a las zonas costeras para realizar actividades recreativas y de esparcimiento. A nivel internacional, las playas de los destinos turísticos más importantes son calificadas por asociaciones como Blue Flag y Tidy Britain Group. Gobiernos trabajan de la mano con estos grupos, para calificar sus destinos turísticos y garantizar así una afluencia de visitantes que buscan calidad en los servicios y en el agua de contacto primario. Estudios realizados por diversas agrupaciones e investigadores hacen asociaciones entre los niveles de contaminación de las aguas de recreo y la aparición de enfermedades (CABELLI, 1983; CHEUNG, 1989, CEPIS, 2000; EPA, 1999; EL SHARKAWI, 1979; FLEISHER, 1996; FERNANDEZ, 1992; FEWTRELL, 1993; HENDERSON, 1968; LIGHTFOOT, 1989; PHLS, 1959; OMS, 1998/2003; PNUMA; CSHP; Blue Flag y Tidy Britain Group, entre otros).

Esta situación hace necesario considerar los factores sociales, culturales, ambientales y económicos del lugar, así como las vías de exposición y la naturaleza y gravedad de 
los peligros. De esta manera, las instituciones responsables de la salud pública a nivel local y nacional podrán desarrollar estándares adecuados para cada región y subregión según las diferencias que existan. (ALCALDÍA DE CARATGENA, 2002)

En Colombia, el Decreto 1594 de 1984 se encarga de establecer los criterios físicoquímicos y microbiológicos mínimos de la calidad de las aguas recreativas, que a continuación se enumeran: (MINISTERIO DE SALUD, 1984)

Artículo 42: Los criterios de calidad admisibles para la destinación del recurso para fines recreativos mediante contacto primario, son los siguientes:

- Coliformes fecales:

$200 \mathrm{NMP} / 100 \mathrm{ml}$

- Coliformes totales:

$1.000 \mathrm{NMP} / 100 \mathrm{ml}$

- Compuestos fenólicos Fenol:

$0.002 \mathrm{mg} / \mathrm{l}$

- Oxígeno disuelto.

70\% concentración de saturación

(concentración mínima de

$O D=4.68 \mathrm{mg} / \mathrm{l}$ en agua salada)

- $p H$ :

$5-9$ unid

- Tensoactivos (SAAM)

0.5 .

Reconociendo la importancia de la calidad de las aguas de recreo, la EPA (Environment Protection Agency) recomendó en 1986 nuevas normas para el monitoreo, que incluyen el uso de $E$. coli y Enterococos (E) como indicadores que reemplacen a los coliformes fecales, ya que se hace necesario desarrollar investigaciones para establecer los indicadores adecuados en zonas Tropicales. (EPA, 1999).

En Cartagena existen fuentes que pueden representar un riesgo de contaminación para las aguas de recreo de contacto primario y secundario. Tales fuentes de contaminación son: Contaminación industrial, específicamente en la Bahía, la zona industrial de Mamonal; el Canal del Dique, por su aporte de residuos y sedimentación; contaminación por la actividad marítima y portuaria, y contaminación por descarga de aguas residuales domésticas. (GERFILA, 1993; IDEADE, 1995)

Dentro de los antecedentes en la zona, se tiene la valoración realizada por Urbina (1994) de la calidad microbiológica de las aguas de recreo de las playas de Castillogrande y el Laguito. En estos estudios se dio a conocer que los niveles de calidad del agua no cumplían con los estándares para coliformes totales (CT) y coliformes fecales (CF) del Decreto 1594 de 1984.

El Instituto de Investigaciones Marinas y Costeras, Invemar (2001) citó que la Bahía de Cartagena y la Ciénaga de Tesca (o Ciénaga de la Virgen en Cartagena), interconectadas por un canal y una serie de lagunas, se ven influenciadas por una contaminación alta de coliformes fecales como consecuencia de las descargas de aguas negras a través del alcantarillado sanitario de la ciudad de Cartagena y de descargas que se hacen de forma directa. De igual forma, la conjugación de las descargas y el régimen de circulación de corrientes de la Bahía y la Ciénaga, favorecen la permanencia de coliformes en niveles no permisibles en algunos sectores turísticos, como Castillo Grande y El Laguito.(CARDIOUE, 1997, 1998; CIOH, 2002; COLCIENCIAS 1999). 
En el presente trabajo se evaluaron los niveles de contaminación de las playas de Bocagrande y de Marbella, de parámetros microbiológicos y físico-químicos, a través del análisis de los agentes microbiológicos coliformes totales, E. coliy enterococos, de los parámetros químicos tensoactivos y fenoles, al igual que de parámetros físicos como $\mathrm{pH}$, oxígeno disuelto y salinidad en los puntos del muestreo. Se realizó el análisis estadístico correspondiente.

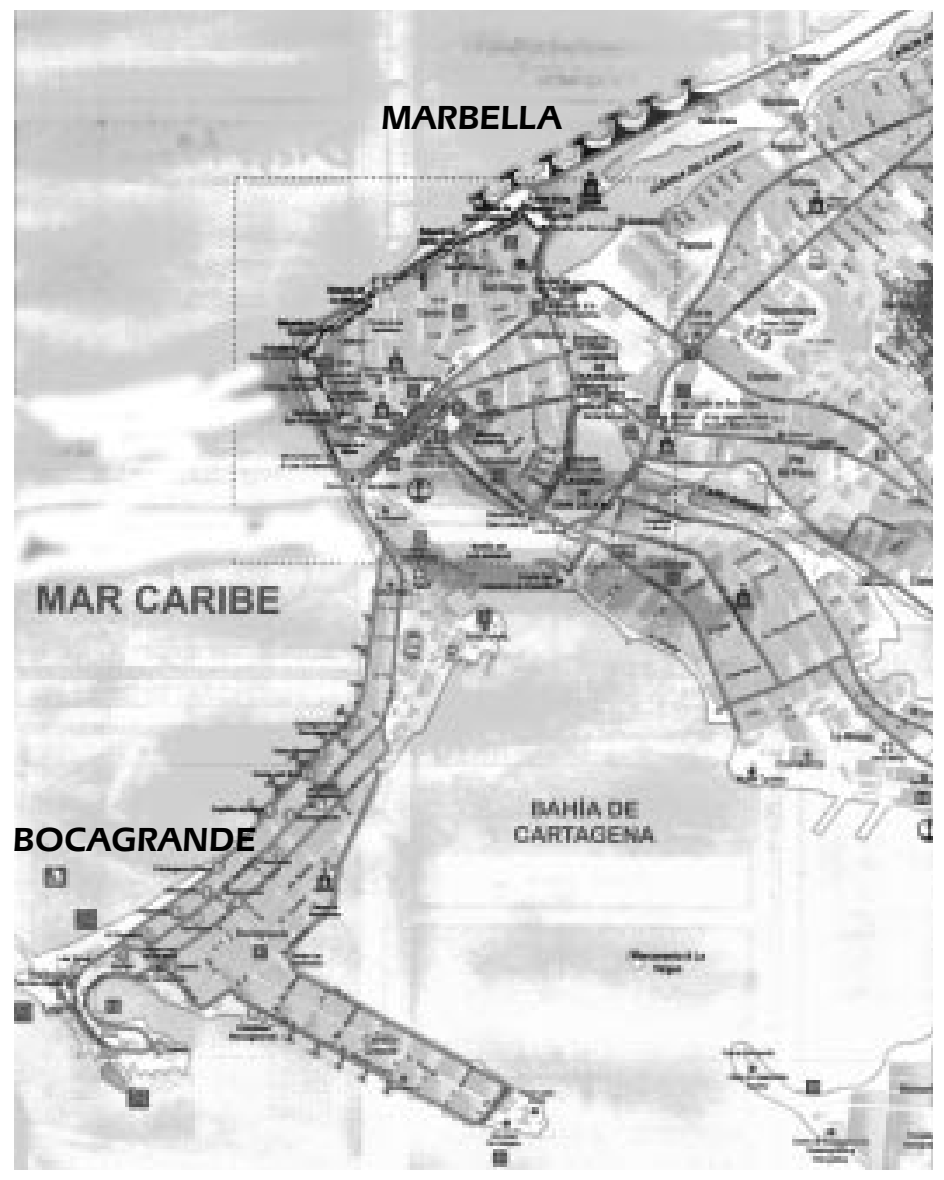

Figura 1: Ubicación de las playas más frecuentadas en Cartagena.

\section{METODOLOGIA}

\subsection{Selección repuntos a muestrear.}

Se seleccionaron las playas de Bocagrande y Marbella por ser las más frecuentadas por los turistas. En cada playa se seleccionaron tres estratos o zonas cercanas a los hoteles, con mayor densidad de visitantes. 
El Método Estratificado Aleatorio (MEA) establece que todas las unidades en la población deben tener la misma probabilidad de ser seleccionadas. Por esta razón se escogieron al azar 2 puntos en cada estrato que cumplieran condiciones de profundidad mínima para tomar la muestra en marea baja y facilidad de acceso a cada punto. Allí se realizaron 9 jornadas de muestreo, es decir, 54 observaciones por playa, por parámetro o variable estudiada.

$$
\begin{aligned}
& n_{h}=\frac{n}{H} \\
& n_{h}=\frac{6}{3}=2
\end{aligned}
$$

$n \quad$ tamaño de la muestra global por población o playa

h estrato

H número total de estratos en cada playa o población

\subsection{Peso o ponderación por estrato.}

El peso o ponderación (W/h) es la representación que cada estrato tiene en cada una de las playas. De esta forma, la ponderación de cada estrato en la playa de Bocagrande aparece en la tabla 2.1 y se calcula así:

$$
W_{h}=\frac{N_{h}}{N}
$$

$W_{\mathrm{h}}$ : peso o ponderación de la población que pertenece al estrato $\mathrm{h}$

$\mathrm{N}_{\mathrm{h}}$ : número de puntos considerados a pertenecer a la muestra del estrato $_{h}$, en una playa en particular

$\mathrm{N}$ : número total de puntos considerados en una playa en particular

Para el estrato 1: $\quad W_{1}=\frac{N_{1}}{N}$

$$
W_{1}=\frac{12}{34}=0.35 * 100=35.3 \%
$$

Tabla 2.1. Peso o ponderación de cada uno de los estratos, en las playas de Bocagrande y Marbella

\begin{tabular}{c|c|c}
\multirow{2}{*}{} & \multicolumn{2}{|c}{ Playa } \\
\cline { 2 - 3 }$W_{1}(\%)$ & Bocagrande & Marbella \\
\hline$W_{2}(\%)$ & 35.3 & 50 \\
\hline$W_{3}(\%)$ & 38.2 & 30 \\
\hline Total & 26.5 & 20 \\
\hline
\end{tabular}

Se tomaron las coordenadas geográficas con el Sistema de Información Geográfico, GPS, para la ubicación de los puntos cada vez que se realizaron los muestreos. 


\subsection{Recolección de muestras.}

Los puntos seleccionados para los muestreos estaban ubicados en zonas de playa de arena, con fácil acceso para tomar la muestra. Las muestras de agua analizada fueron tomadas en época seca, entre el 9 de febrero de 2004 y el 14 de abril de 2004. Las muestras de agua se emplearon para dos tipos de análisis: análisis in situy para análisis de laboratorio, estos últimos llevados a cabo en el laboratorio de Ingeniería Ambiental del Instituto de Ensayos e Investigaciones de la Universidad Nacional de Colombia en Bogotá.

Las muestras se recogieron siguiendo las indicaciones de Standard Methods for Recreational Waters (SM 9213 D) que se describen a continuación: (APHA, 1992)

Las muestras de agua se recolectaron en recipientes de vidrio con tapa plástica, limpios y esterilizados. La profundidad de recolección fue de aproximadamente $30 \mathrm{~cm}$, tratando de no recoger sedimentos. Esto no se cumplió en algunas ocasiones, ya que la rompiente de las olas era muy fuerte y la arena entraba en la botella. Dos días por semana se realizó la recolección, entre las 8:00 y las 10:00 am, un día en la playa Bocagrande y al otro en la playa Marbella. Una vez rotuladas las botellas y empacadas correctamente para el transporte, se colocaron en el servicio de correo aéreo en Cartagena, hacia Bogotá.

\subsection{Análisis de laboratorio}

En el Laboratorio de Ingeniería Ambiental del Instituto de Ensayos e Investigaciones de la Universidad Nacional, en Bogotá, se realizaron los análisis microbiológicos Coliformes Totales, CT; E. coli, E.coli; Enterococos, E; y las pruebas químicas Fenoles, F; Tensoactivos aniónicos, $T$

\subsection{Análisis in situ.}

Las mediciones de los parámetros físicos temperatura, $\mathrm{pH}$, salinidad y oxígeno disuelto se realizaron in situ. Los electrodos se calibraban antes de iniciar las jornadas de muestreo. El electrodo se introdujo a $30 \mathrm{cms}$ de profundidad en el agua del mar, hasta que la lectura en la pantalla se estabilizara.

\section{RESULTADOS Y ANÁLISIS DE RESULTADOS}

\subsection{Descripción de la zona de estudio.}

Se observó que las playas de Bocagrande constituyen la zona turística de mayor importancia de Cartagena y reciben mantenimiento diario, que consiste en la recolección de permanente de basuras y el barrido de la arena. Se instalan canecas para que los usuarios depositen los residuos sólidos. En las playas de Marbella no hay actividades de mantenimiento.

En ambas playas es común ver mascotas (perros) y caballos defecando. También fue frecuente ver aves y otros animales que se alimentaban de los residuos orgánicos que dejan los usuarios de las playas. 


\subsection{Metodología estadística empleada}

Se aplicó el análisis estadístico exploratorio de Series de Tiempo, seguido de un Análisis Estadístico Clásico, con el fin de encontrar la mejor metodología estadística para el procesamiento de los datos. Complementariamente, a los resultados de los parámetros microbiológicos, se les realizó un análisis descriptivo de Gráficas de Probabilidad.

\subsection{Verificación de las condiciones ambientales a través del tiempo.}

El análisis estadístico exploratorio de series de tiempo nos permite determinar si existe un patrón o tendencia dependiente del tiempo. La observación de las condiciones ambientales a través del tiempo es una prueba útil para verificar si es o no necesaria la consideración del análisis de series de tiempo (Universidad Nacional, asesoría personall).

Se realizaron gráficos exploratorios que muestran el comportamiento de los datos a través del tiempo. Los gráficos 3.1 a 3.4 reúnen la información de ambas playas, con un total de 54 observaciones por cada una de ellas recolectadas durante 9 semanas en seis puntos de muestreo.

Como puede observarse en los gráficos 3.1 a 3.4, las variables ambientales no muestran un comportamiento estacionario o con tendencia durante las semanas de seguimiento, por lo que no se hace necesario el análisis de series temporales considerando las condiciones ambientales. En los gráficos 3.5 se observa las series de tiempo para los parámetros microbiológicos.

Gráficos 3.1 a 3.4: Serie de tiempo de los parámetros físicos $\mathrm{pH}, \mathrm{OD}$, 't y $\mathrm{S}$ en las playas de Bocagrande y Marbella.
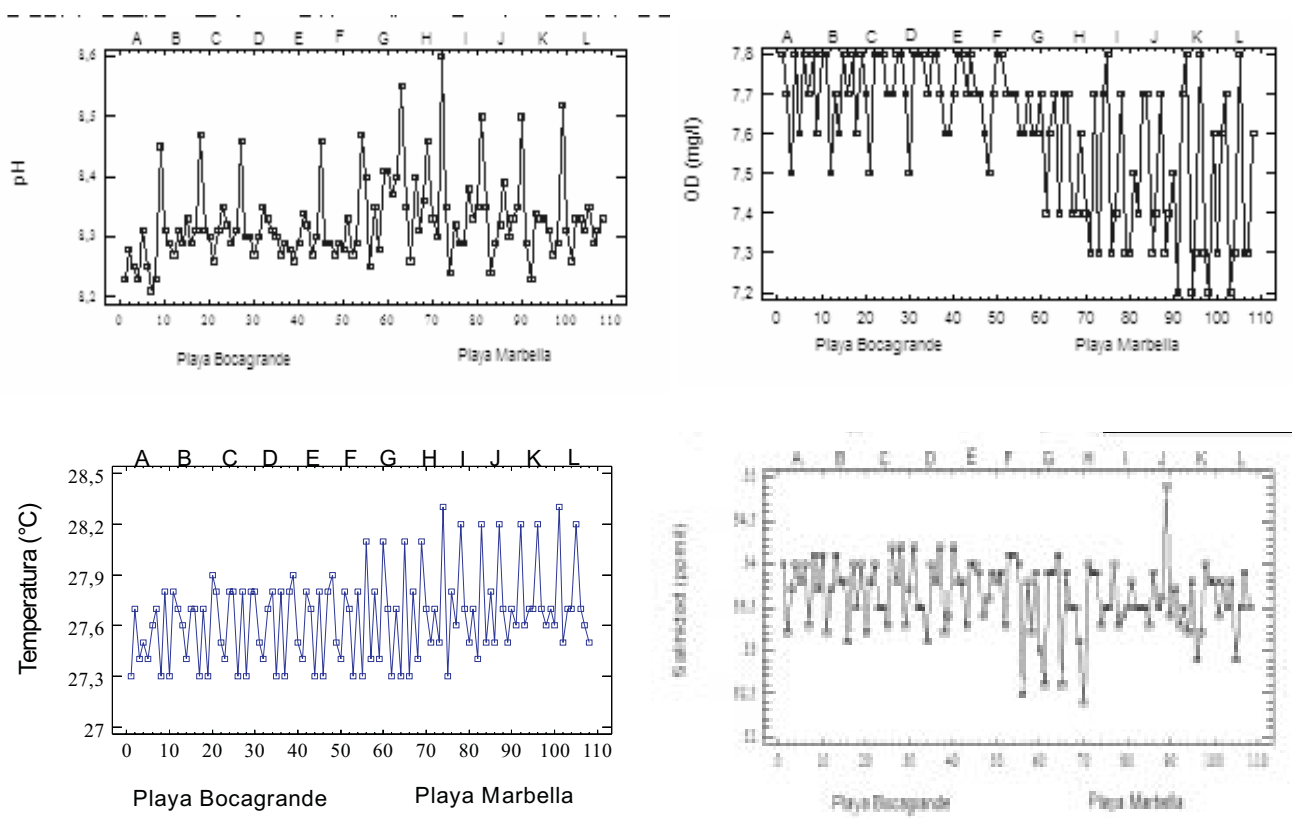
Gráficos 3.5: Serie de tiempo de los parámetros microbiológicos en las playas de Bocagrande (columna izquierda) y de Marbella (clna derecha)
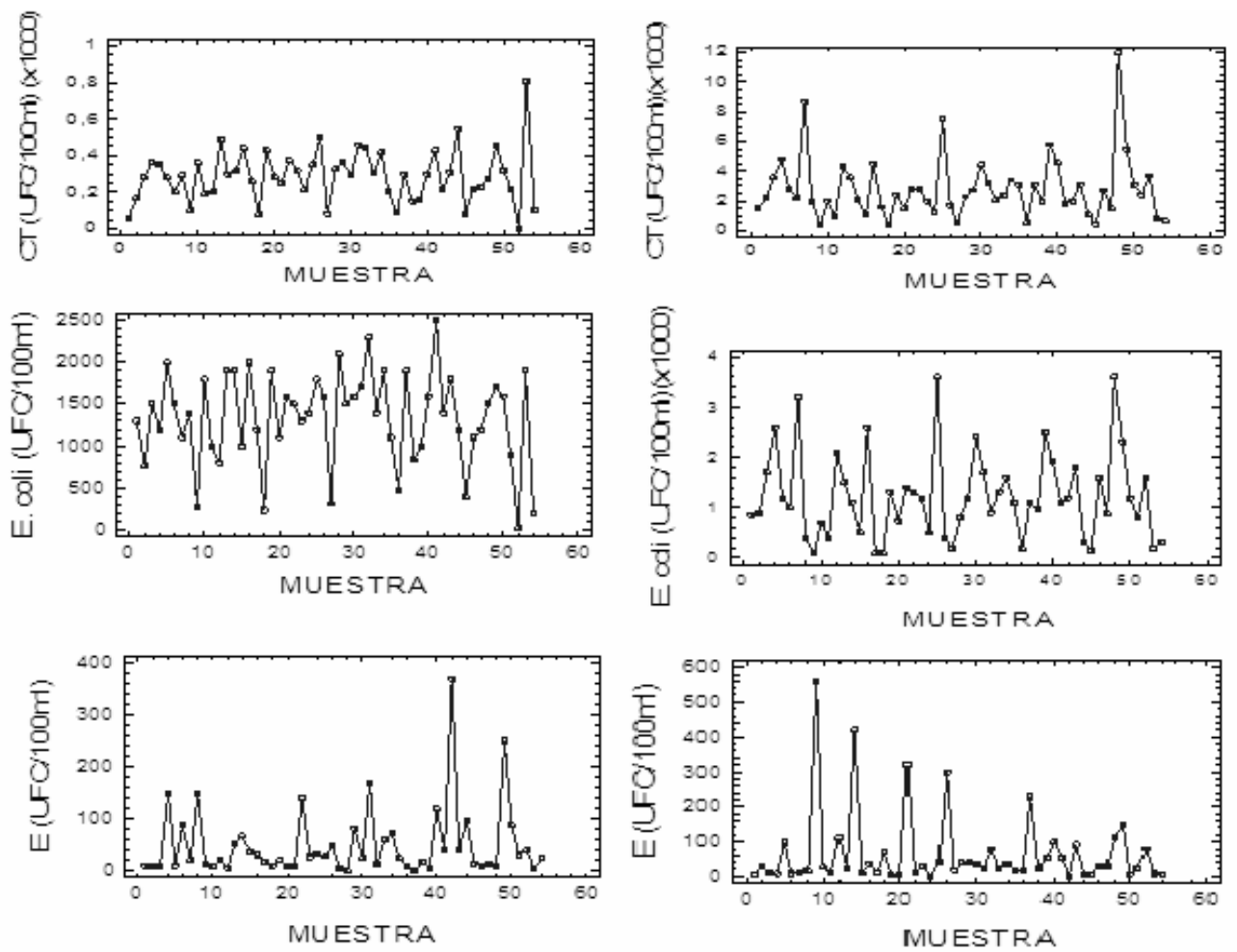

Tanto los parámetros microbiológicos como los físicos muestran un comportamiento estacionario durante las semanas de seguimiento. Por esta razón, se procedió a realizar un análisis estadístico clásico para los parámetros físicos y microbiológicos, a través de un análisis descriptivo y de un análisis comparativo de los promedios.

Como también se observó un comportamiento ligeramente distinto por playa, se decidió analizar separadamente los datos de cada una de ellas.

\subsection{Análisis Clásico: Estimación del promedio para las variables de estudio.}

3.4.1 Parámetros microbiológicos.

A continuación se presentan los resultados de los promedios geométricos para cada uno de los parámetros microbiológicos, teniendo en cuenta los puntos y estratos parametrados en la playa de Bocagrande y de Marbella. 
Tabla 3.1: Promedios geométricos para los parámetros microbiológicos por punto, para la playa de Bocagrande.

\begin{tabular}{c|c|c|c|c} 
Estrato & Punto & $\begin{array}{c}\text { CT } \\
\text { (UFC/100 ml) }\end{array}$ & $\begin{array}{c}\text { E. Coli } \\
\text { (UFC/100 ml) }\end{array}$ & $\begin{array}{c}\text { Enterococos } \\
\text { (UFC/100 ml) }\end{array}$ \\
\hline 1 & Punto A & 2364 & 1088 & 28 \\
\hline 1 & Punto B & 2506 & 1260 & 24 \\
\hline 2 & Punto C & 2813 & 1262 & 21 \\
\hline 2 & Punto D & 2875 & 1377 & 38 \\
\hline 3 & Punto E & 2395 & 1250 & 40 \\
\hline 3 & Punto F & 1587 & 627 & 28 \\
\hline
\end{tabular}

Tabla 3.2: Promedios para los parámetros microbiológicos por punto, para la playa de Marbella.

\begin{tabular}{c|c|c|c|c}
\hline Estrato & Punto & $\begin{array}{c}\text { CT } \\
\text { (UFC/100 ml) }\end{array}$ & $\begin{array}{c}\text { E. Coli } \\
\text { (UFC/100 ml) }\end{array}$ & $\begin{array}{c}\text { Enterococos } \\
\text { (UFC/100 ml) }\end{array}$ \\
\hline 1 & Punto G & 2352 & 930 & 22 \\
\hline 1 & Punto H & 1776 & 586 & 37 \\
\hline 2 & Punto I & 1993 & 864 & 20 \\
\hline 2 & Punto J & 2392 & 1062 & 30 \\
\hline 3 & Punto K & 2143 & 926 & 26 \\
\hline 3 & Punto L & 2491 & 950 & 22 \\
\hline
\end{tabular}

Tabla 3.3: Promedios ponderados' para los parámetros microbiológicos por estrato, para la playa de Bocagrande

\begin{tabular}{l|c|c|c|c} 
Estrato & $\begin{array}{c}\text { Peso } \\
\text { Wh }\end{array}$ & $\begin{array}{c}\text { CT } \\
\text { (UFC/100 ml) }\end{array}$ & $\begin{array}{c}\text { E. coli } \\
\text { (UFC/100 ml) }\end{array}$ & $\begin{array}{c}\text { Enterococos } \\
\text { (UFC/100 ml) }\end{array}$ \\
\hline 1 & 35 & 859 & 379 & 9 \\
\hline 2 & 38 & 1087 & 504 & 11 \\
\hline 3 & 27 & 515 & 234 & 9 \\
\hline Promedio estimado para la playa & 2461 & 1117 & 29 \\
\hline Ancho del Intervalo de confianza & 133 & 107 & 4 \\
\hline Limite inferior & 2328 & 1010 & 25 \\
\hline Limite superior & 2594 & 1224 & 33 \\
\hline Coeficiente de variación CV (\%) & 2 & 4 & 5 \\
\hline
\end{tabular}

Los promedios ponderados o estimados tienen en cuenta el peso o ponderación de cada estrato. 
El promedio estimado se obtiene multiplicando el promedio geométrico por la ponderación de cada estrato. El ancho del intervalo de confianza nos muestra el grado de validez de las estimaciones que se hacen. Con el promedio estimado y el ancho del intervalo de confianza se obtienen los límites superior (sumándolos) e inferior (restándolos). En el Anexo 1, se encuentran los procedimientos para calcular el CV para CT en la playa de Bocagrande. Los coeficientes de variación CV obtenidos garantizan la poca variabilidad de los datos y nos permiten comparar los intervalos de confianza de ambas playas.

Tabla 3.4: Promedios ponderados para los parámetros microbiológicos por estrato, para la playa de Marbella.

\begin{tabular}{l|c|c|c|c} 
Estrato & $\begin{array}{c}\text { Peso } \\
\text { Wh }\end{array}$ & $\begin{array}{c}\text { CT } \\
\text { (UFC/100 ml) }\end{array}$ & $\begin{array}{c}\text { E. coli } \\
\text { (UFC/100 ml) }\end{array}$ & $\begin{array}{c}\text { Enterococos } \\
\text { (UFC/100 ml) }\end{array}$ \\
\hline 1 & 50 & 1022 & 369 & 15 \\
\hline 2 & 30 & 655 & 287 & 7 \\
\hline 3 & 20 & 462 & 188 & 5 \\
\hline \multicolumn{2}{l|}{ Promedio estimado para la playa } & 2138 & 844 & 27 \\
\hline \multicolumn{2}{|l|}{ Ancho del Intervalo de confianza } & 162 & 88 & 4 \\
\hline \multicolumn{2}{l|}{ Limite inferior $\quad 1977$} & 756 & 22 & \\
\hline \multicolumn{2}{l|}{ Limite superior 2300} & 932 & 31 & \\
\hline \multicolumn{2}{l|}{ Coeficiente de variación CV (\%) } & 3 & 4 & 6 \\
\hline
\end{tabular}

Como se puede observar en la gráfica 3.6, el límite superior-superior de los niveles de CT se encontró en la playa de Bocagrande y oscila alrededor de 2600 UFC/100 ml. El límite inferior-inferior se encontró en la playa de Marbella.

Gráfica 3.6: Intervalos de confianza para CT en los estratos muestreados de las playas de Bocagrande y Marbella

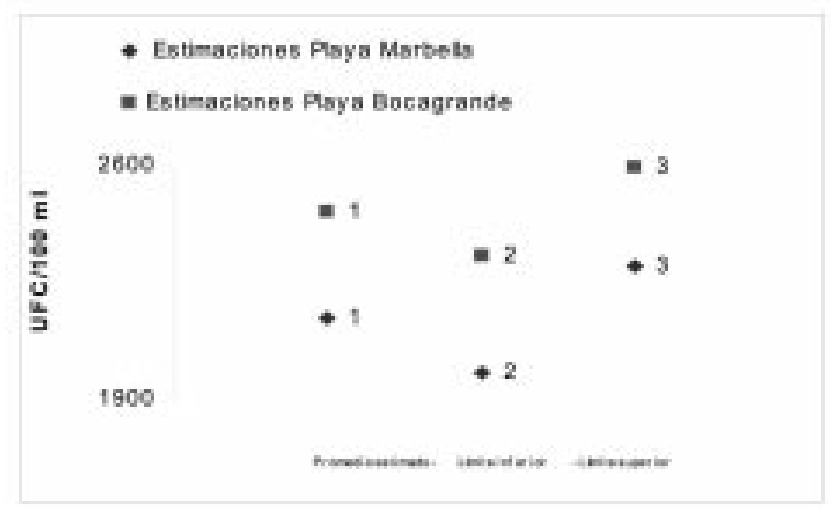

Los niveles de contaminación microbiológica se pueden relacionar con el impacto que animales como aves, perros, caballos. En las jornadas de muestreo se observaron animales usando las playas junto con bañistas (Figura 3.1.) 
Tabla 3.5: Calidad del agua marina con el riesgo relativo RR natación vs no natación en aguas de diferente calidad.(OMS, 1998)

\begin{tabular}{|c|c|c|c|c|c|}
\hline Autor año & $\begin{array}{l}\text { Efectos } \\
\text { sobre la salud }\end{array}$ & Ind. & $\begin{array}{l}\text { Recuento de } \\
\text { indicadores } \\
\text { promedio } \\
\text { (/100ml) }\end{array}$ & $\begin{array}{l}\text { RR } \\
\text { (95\% de } \\
\text { confiabilidad) }\end{array}$ & $\begin{array}{l}\text { Tasas de } \\
\text { incidencia } \\
(\mathrm{TI} * 1000)\end{array}$ \\
\hline Bandaranayake, 1995 & GEAC & E & 232 vs 1.5 & $0.84(0.27-2.63)$ & 22 vs 262 \\
\hline Cabelli, 1982 & G & $E$ & 154 vs 35 & $2.21(1.41-3.47)^{\star *}$ & 60 vs 27 \\
\hline Cabelli Egipto, 1983 & Vómito Diarrea & E & 286 vs 103 & $0.97(0.38-2.51)$ & 16 vs 16 \\
\hline Cheung, 1989 & GEAC & E coli & 269 vs 69 & $1.75(0.54-5.69)$ & 6.5 vs 2.1 \\
\hline Fattal, 1983 & Gl Edad 0-4 & E & $\begin{array}{l}49(25-410) \text { vs } \\
(0-14)\end{array}$ & $1.88(1.01-3.5)^{\star}$ & 209 vs 111 \\
\hline $\begin{array}{l}\text { PNUMAVOMS MAPA } \\
\text { N. 20, } 1987\end{array}$ & $\begin{array}{l}\text { Edad 0-4 } \\
\text { GEAC }\end{array}$ & E & $\begin{array}{l}49(25-410) \text { vs } \\
(0-14\end{array}$ & $2.07(1.17-3.65) * *$ & 221 vs 107 \\
\hline $\begin{array}{l}\text { PNUMAVOMS MAPA } \\
\text { N. } 46,1991\end{array}$ & $\begin{array}{l}\mathrm{Gl} \\
\mathrm{R}\end{array}$ & $E$ & $\begin{array}{l}40(31-51) \text { vs } \\
9(2-30)\end{array}$ & $\begin{array}{l}1.95(1.08-3.52)^{*} \\
2.55(1.27-5.05)^{* *}\end{array}$ & $\begin{array}{l}131 \text { vs } 65 \\
124 \text { vs } 47\end{array}$ \\
\hline
\end{tabular}

Clave: ${ }^{*} p<0,05 ;{ }^{* *} p<0,01$

$\mathrm{Gl}=$ síntomas gastrointestinales; $\mathrm{GEAC}=$ gastroenteritis de alta credibilidad $\mathrm{E}$ : enterococos; $\mathrm{R}=$ enfermedades respiratorias

Nota: No se han enumerado todos los resultados que no son significativos

Los niveles de contaminación microbiológica obtenidos en este estudio coinciden con los resultados obtenidos por Urbina (1994), en el hecho de mostrar que los niveles encontrados son superiores a la norma.

Figura 3.1: Bañistas y animales empleando las aguas de recreo simultáneamente.

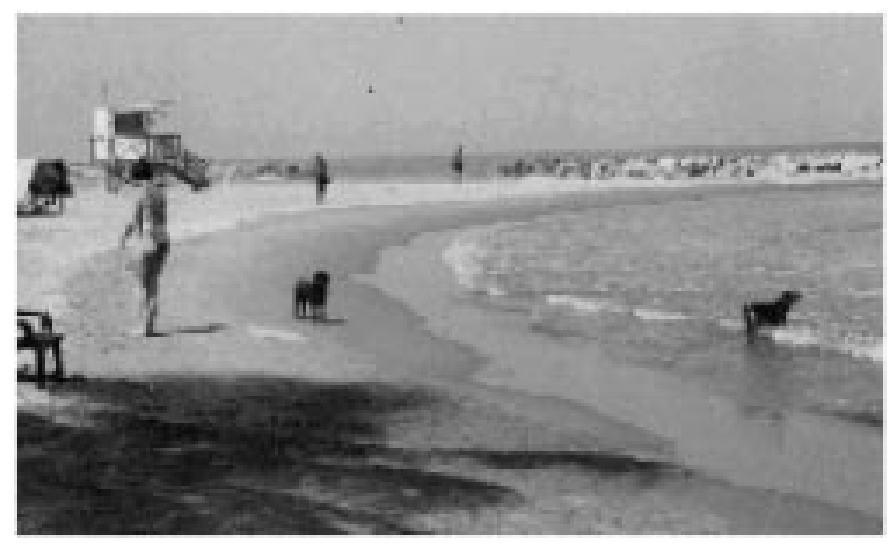

Para a las variables $E$. coli y Enterococos $E$ como parámetros de referencia se tomaron los datos citados por la Organización Mundial de la Salud OMS, de trabajos que correlacionan concentraciones de microorganismos con enfermedades en zonas subtropicales, debido a la carencia de trabajos reconocidos en zonas del Trópico. 


\subsubsection{Parámetros físicos.}

Los parámetros físicos considerados en el Decreto 1594 de 1984 son OD y pH. A continuación se listan los promedios obtenidos en cada punto, estrato y playa, para cada una de estas variables.

El valor admisible en este Decreto está dado en términos de Porcentaje de saturación del $70 \%$ de oxígeno disuelto OD, lo que significa un valor mínimo de 4.48 mg/l para agua salada, a $1 \mathrm{~atm}$, a $27.6^{\circ} \mathrm{C}$ de temperatura. El valor admisible para pH está entre 5 y 9 unidades para aguas de contacto primario. (CHAPRA, 1997; THOMANN, 1987)

Tabla 3.6: Promedios para los parámetros físicos por punto, para la playa de Bocagrande.

\begin{tabular}{l|c|c|c} 
Estrato & $\begin{array}{c}\text { Peso W/h } \\
(\%)\end{array}$ & $\begin{array}{c}\mathrm{OD} \\
(\mathrm{mg} / \mathrm{l})\end{array}$ & $\begin{array}{c}\mathrm{pH} \\
\text { (unidades) }\end{array}$ \\
\hline 1 & 35 & 2,6 & 2,9 \\
\hline 2 & 38 & 3,0 & 3,2 \\
\hline 3 & 27 & 2,1 & 2,2 \\
\hline \multicolumn{2}{l|}{$\begin{array}{l}\text { Promedio estimado } \\
\text { para la playa }\end{array}$} \\
\hline
\end{tabular}

De acuerdo a los resultados obtenidos en las playas de Bocagrande y Marbella, podemos observar que los valores obtenidos para OD y pH se encuentran cumpliendo la normatividad vigente. (Tablas 3.6 y 3.7 )

Tabla 3.7: Promedios para los parámetros físicos por punto, para la playa de Marbella.

\begin{tabular}{|c|c|c|c|}
\hline Estrato & $\begin{array}{c}\text { Peso Wh } \\
(\%)\end{array}$ & OD & $\mathrm{pH}$ \\
\hline 1 & 50 & 3,8 & 4,2 \\
\hline 2 & 30 & 2,3 & 2,5 \\
\hline 3 & 20 & 1,5 & 1,7 \\
\hline \multicolumn{2}{|c|}{ Promedio estimado } & & \\
\hline para la & 7,6 & 8,4 & \\
\hline
\end{tabular}

\subsubsection{Parámetros químicos}

Los análisis de fenoles y de SAAM se realizaron solamente en la semana 1 y en la semana 9 , en los 12 puntos muestreados de las dos playas, ya que los resultados mostraban concentraciones muy bajas o por debajo del límite de detección (Tablas 3.8 y 3.9), lo que coincide con los resultados históricos reportados por CARDIOUE (1997). Esta situación no ameritaba la realización de todos los análisis para las 9 semanas de muestreo. 
Tabla 3.8: Resultados promedio para los parámetros químicos fenoles y tensoactivos, en los puntos muestreados en la playa de Bocagrande.

\begin{tabular}{c|c|c} 
& FENOLES $(\mathrm{mg} / \mathrm{l})$ & SAAM $(\mathrm{mg} / \mathrm{l})$ \\
\hline $\mathrm{A}$ & $<0,02$ & 0,06 \\
\hline $\mathrm{B}$ & $<0.02$ & 0,05 \\
\hline $\mathrm{C}$ & $<0.02$ & 0,055 \\
\hline $\mathrm{D}$ & $<0.02$ & 0,05 \\
\hline $\mathrm{E}$ & $<0.02$ & 0,06 \\
\hline $\mathrm{F}$ & $<0.02$ & 0,05
\end{tabular}

La normatividad colombiana, Decreto 1594 de 1984, exige que los criterios de calidad admisibles para aguas de contacto primario, con respecto a los parámetros químicos, sea en compuestos fenólicos menor a $0.002 \mathrm{mg} / \mathrm{l}$ y en sustancias activas al azul de metileno menor a $0.5 \mathrm{mg} / \mathrm{l}$.

Los promedios obtenidos nos muestran en fenoles niveles inferiores a $<0.02 \mathrm{mg} / \mathrm{l}$. Para tensoactivos en la playa de Bocagrande se obtuvo un promedio de 0.054 mg/l y para la playa de Marbella de 0.056 mg/l. Estos valores nos permiten descartar contaminación de tipo industrial en dichas playas.

Tabla 3.9: Resultados promedio para los parámetros químicos fenoles y tensoactivos, en los puntos muestreados en la playa de Marbella

\begin{tabular}{c|c|c} 
& FENOLES $(\mathrm{mg} / \mathrm{l})$ & SAAM $(\mathrm{mg} / \mathrm{l})$ \\
\hline $\mathrm{G}$ & $<0.02$ & 0,06 \\
\hline $\mathrm{H}$ & $<0.02$ & 0,045 \\
\hline $\mathrm{I}$ & $<0.02$ & 0,06 \\
\hline $\mathrm{J}$ & $<0.02$ & 0,06 \\
\hline $\mathrm{K}$ & $<0.02$ & 0,055 \\
\hline $\mathrm{L}$ & $<0.02$ & 0,055 \\
\hline
\end{tabular}

\subsection{Análisis descriptivo}

Con respecto a los resultados obtenidos en Enterococos y E. coli, podemos inferir a partir de las referencias bibliográficas citadas (Tabla 3.4) que existe un riesgo relativo RR de contraer enfermedades al estar en contacto con aguas de recreo contaminadas por estos microorganismos. Estas enfermedades pueden ser gastrointestinales, respiratorias, vómito y diarrea, con diferente riesgo relativo RR, de acuerdo al recuento y al tipo de exposición.

Los valores correspondientes a coliformes totales se ajustaron mejor al gráfico de probabilidad normal (Gráfica 3.7). Allí se puede apreciar que los promedios obtenidos, al igual que las concentraciones de $E$. coli, son mayores que el valor de referencia. Para Enterococos $E$ podemos observar que el $94 \%$ y $93 \%$ del total de los valores observa- 
dos para Bocagrande y Marbella, respectivamente, son iguales o inferiores a los reportados por Bandarayake (1995) relacionados con gastroenteritis de alta credibilidad.

Gráfica 3.7: Gráficos de probabilidad normal, log-normal y exponencial para coliformes totales CT, E. coli y Enterococos en las playas de Bocagrande (izq) y Marbella (der)
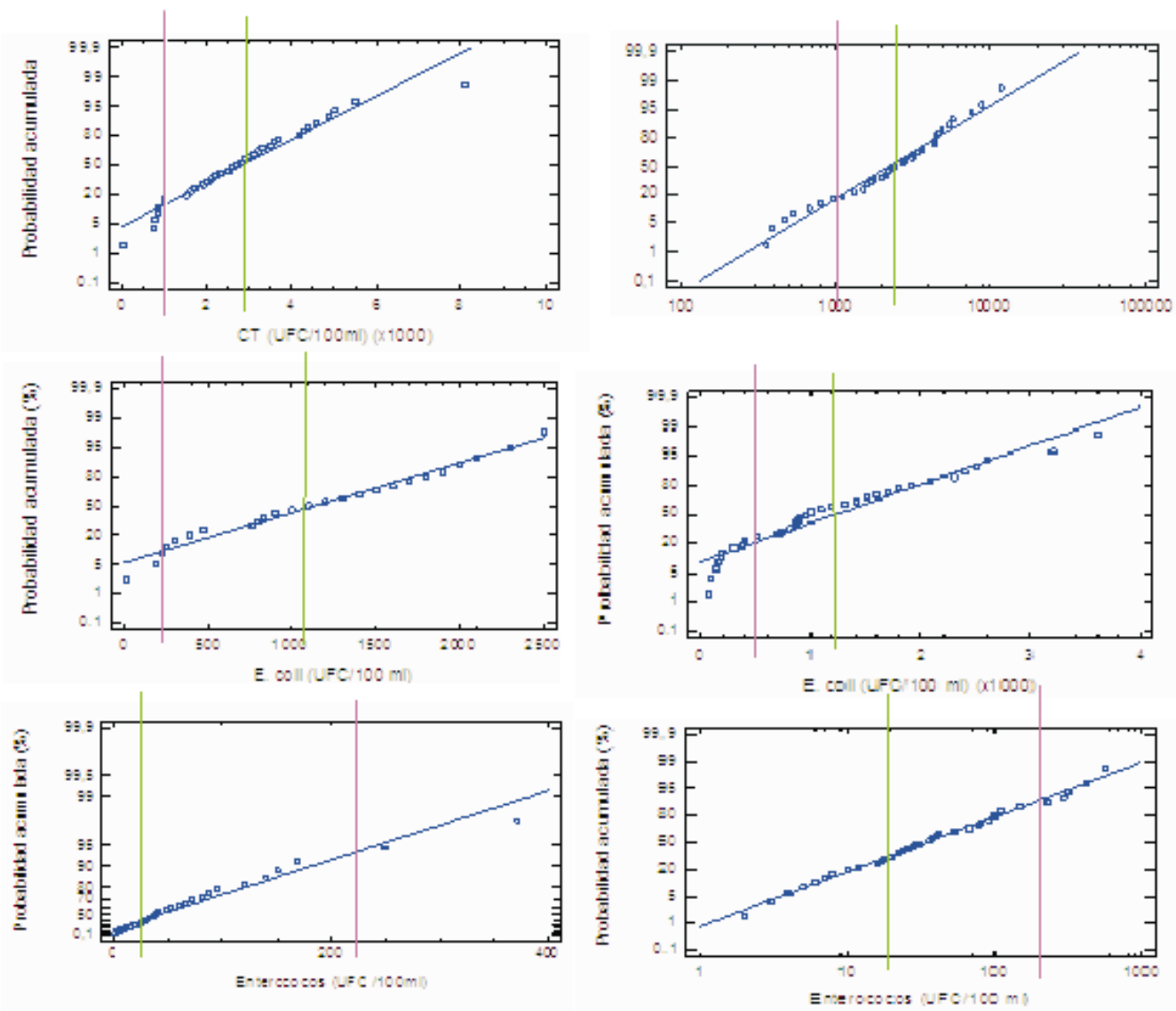

Valores de referencia:

- CT $=1000$ UFC/100 ml, valor permisible del Decreto 1594 de 1984

$\mathrm{E}$. coli $=269$ UFC/100 ml , (Cheung, 1989: tasa de incidencia de 6.5 casos por mil de gastroenteritis aguda)

Enterococos $=232$ UFC/100 ml, (Bandaranayake, 1995: t tasa de incidencia de 22 casos por mil de gastroenteritis aguda)

Promedios geométricos obtenidos para las playas 


\section{CONCLUSIONES}

De acuerdo a los resultados obtenidos para cada una de las variables analizadas, tenemos que en general los estratos evaluados cumplen con la normatividad vigente para aguas de recreo de contacto primario, ya que las concentraciones obtenidas fueron inferiores a lo dispuesto por el Decreto 1594 de 1984.

Los parámetros físicos pH y OD evaluados, cumplieron con suficiencia los estándares que contempla la norma colombiana. Los parámetros químicos evaluados en este trabajo, tensoactivos (SAAM) y fenoles, presentaron promedios de concentraciones inferiores a las que regula el Decreto 1594 de 1984, razón por la cual se descarta un impacto industrial causado por estos agentes en las playas de Bocagrande y Marbella.

Con respecto a las variables microbiológicas, se observó que las aguas de recreo de las playas evaluadas presentan mayores niveles de contaminación por coliformes totales $C T$, seguidos de E. coliy, finalmente Enterococos $\mathrm{E}$, en orden descendente de concentración. Se sugiere realizar análisis microbiológicos en áreas de playa "rural", cercana a Cartagena, para determinar si los resultados aquí obtenidos serían concentraciones base para las aguas de recreo de las playas de esta zona geográfica. Es importante conocer fotografías satelitales del área de estudio para evaluar la posible correlación del comportamiento de las corrientes con los niveles de contaminación microbiológica obtenidos.

Aproximaciones a la calidad sanitaria de aguas de recreo de Cartagena, como la que se presenta en este trabajo, permite a la comunidad académica, a los gobernantes y empresarios del sector del turismo emprender un frente común que beneficie al buen nombre de la ciudad como destino turístico, estableciendo los lineamientos para garantizar y satisfacer las demandas internacionales que buscan los turistas.

De igual forma, se recomienda realizar monitoreos con mayor frecuencia, por lo menos de los parámetros exigidos por el Decreto 1594 de 1984, ya que las playas son visitadas durante todo el año.

\section{BIBLIOGRAFÍA}

ACUACAR: Estimación de caudales y calidad de agua. 1996. Sin publicar.

ALCALDÍA DE CARTAGENA: Perfil epidemiológico de Cartagena. 2002. Oficina de Planeación DADIS- VSP DADIS

ALCALDÍA DE CARTAGENA: Plan de Ordenamiento Territorial de Cartagena de Indias. Síntesis del diagnóstico. 2001

APHA. Standard Methods for tha examination of water and watewater USA. 1992.

BANDARANAYAKE DR, Turner SJ, McBride GB, Lewis GD, Till DG. HealthEffects of Bathing at Selected New Zealand Marine Beaches. 1995 Nueva Zelanda.

CABELLI VJ: Health effects criteria for marine recreational waters. 1983. Research Triangle Park, EPA, EPA-600/1-80-031, 98pp. 
CARDIQUE, UDC: Plan de manejo y control ambiental de las zonas portuarias y costeras en el área de jurisdicción de CARDIOUE. 1998. Tomo I, II, III.

CARDIQUE, $\mathrm{ClOH}$. Caracterización y diagnóstico de la zona costera. 1997.Tomo I, II, III.

CHAPRA, Steven: Surface water-quality modeling. Mc Graw Hill International Editions. 1997. Singapure.

CHEUNG, W.H.S., Hung, R.P.S. Chang, K.C.K. y Kleevens, J.W.L., Epidemiological study of beach water pollution and health-related bathing water standards in Hong Kong. Water Science and Technology. 1989. 23/1-3/, 243-252.

CHEUNG WHS, Chang KCK, Hung RPS: Variations in microbial indicator densities in beach water and health-related assessment of bathing water quality. Epidemiol Infect. 1991, 106, 329-344.

CEPIS: Guía para ambientes seguros en aguas recreativas. 1998.

$\mathrm{ClOH}$ : Estudio del comportamiento de la Contaminación Bacteriana en la Bahía de Cartagena. 2002. Boletín $\mathrm{ClOH}$ N. 20.

COLCIENCIAS, Plan Estratégico 1999-2004: Contaminación de playas y de aguas de balnearios turísticos. Contaminación de la cuenca sur del Caribe por descargas del Río Magdalena. Programa Nacional de Ciencia y Tecnología del Mar.

CSHP CONSEIL SUPERIEUR D' HYGIÈNE PUBLIQUE DE FRANCE: Qualité microbiologique des sables: essai d' un nouveau précédé de désinfection.1990.

EL SHARKAWI F, HASSAN MNER. 1979 The relation between the state of pollution in Alexandria swimming beaches and the occurrence of typhoid among bathers. Bulletin of the High Institute of Public Health Alexandria, IX, 337-351.1979

EPA: Ambient Water Quality Criteria for Bacteria. 1986. Washington, DC

EPA: Action Plan for Beaches. 1999. Washington DC

EPA: Improved Enumeration Methods for the Recreational Water Quality Indicators: Enterococci and E.coli. 2000. Washington DC

FLEISHER JM, Kay D, Salmon RL, Jones F, Wyer MD, Godfree AF. Marine waters contaminated with domestic sewage: nonenteric illnesses associated with bather exposure in the United Kingdom. 1996. American Journal of Public Health, 86(9): 1228- 1234.

FEWTRELL y otros: Pathogens in surface temperate waters. Samara Press, 1993. Reino Unido, 262pp.

GEFIRLA/93/G4: Monitoreo de la calidad ambiental en la Bahía de Cartagena y la Zona Industrial de Mamonal.

HENDERSON, J. M. Enteric Disease Criteria for Recreational Waters. 1968. J. San. Eng. Div. 94:1253.

IDEADE Cartagena, Ambiente y desarrollo. Lineamientos para el Ordenamiento Terrotorial. 1995. 
INVEMAR. Diagnóstico y evaluación de la Calidad Ambiental Marina en el Caribe y Pacífico Colombiano. Red de Vigilacia para la Conservación y Protección de las aguas Marinas y Costeras de Colombia. 2001.

MADIGAN, MARTINKO, PARKER. Brock Biología de los microorganismos. 1997. Prentice Hall. España

MINISTERIO DE SALUD: Decreto 1594 de 1984. Por el cual se reglamenta parcialmente el Título I de la Ley 9 de 1979, así como el Capítulo II del Título VI -Parte III- Libro II y el Título III de la Parte III -Libro I-del Decreto - Ley 2811 de 1974 en cuanto a usos del agua y residuos líquidos.

MOORE, B. 1975. The Case Against Microbial Standards for Bathing Beaches. In: International Symposium on Discharge of Sewage From Sea Outfalls. Ed. A. L. H. Gameson. Pergamon Press, London. p. 103.

OMT: Informa anual. 1995

OMS/PNUMA 1994. Microbiological quality of coastal recreational water. MED POL Phasell, Informe sobre una reunión conjunta entre OMS/PNUMA, 9 al 12 de junio de 1993, Atenas, Grecia, pub. Oficinal regional de la OMS para Europa, 1994.

OMS-OPS: Guía para ambientes seguros en aguas recreativas. 1998. En: Volumen 1. Versión Preliminar.

OMS: Guidelines for safe recreational water environments. Volumen 1: Coastal and fresh water. 2003

OMS (2003-2): http://www.consumaseguridad.com/web/es/normativa_legal/2003/ 10/2 0/8908.php

PNUMA/OMS: Epidemiological Studies Related to Environmental Quality Criteria for Bathing Waters, Shellfish-Growing Waters and Edible Marine Organisms (Activity D). 1988. Informe final de un proyecto sobre la relación entre la calidad microbiológica de las aguas marinas y costeras y los efectos sobre la salud. pub. Programa de las Naciones Unidas para el Medio Ambiente; MAP Technical Report Series 20, Atenas, Grecia, 156pp.

PRUSS, A. 1998 A review of epidemiological studies from exposure to recreational water, International Journal of Epidemiology, 27, 1-9

PHLS Public Health Laboratory Service: Sewage contamination of coastal bathing waters in England and Wales: a bacteriological and epidemiological study. Journal of Higiene 43, 435-472. 1959

THOMANN, ROBERT: Principles of Surface Water Quality Modeling and Control. Harper Collins Publishers. 1987

URBINA, DELFINA. Reflexiones sobre la contaminación de la Bahía de Cartagena y Área de Influencia. 1994. Unicarta. Cartagena. 
Anexo 1: Cálculo del CV para CT en la playa de Bocagrande.

1. Promedio estimado para la playa

$$
\begin{aligned}
& \bar{x}=\sum_{h=1}^{3} W_{h} \bar{x}_{E h} \\
& \bar{x}=(0.35 * 2434)+(0.38 * 2844)+(0.26 * 1944) \\
& \bar{x}=2461 U F C / 100 m l
\end{aligned}
$$

Para el calculo de la varianza de en el estrato 1:

$$
\begin{aligned}
\operatorname{var}_{E h} & =\frac{\sum_{h=1}^{N}\left(x_{E h}-\bar{x}_{E h}\right)^{2}}{n_{h}-1} \\
\operatorname{var}_{E 1} & =\frac{(2364-2434)^{2}+(2506-2434)^{2}}{2-1} \\
\operatorname{var}_{E 1} & =10082
\end{aligned}
$$

La varianza de cada punto:

$$
\begin{aligned}
& \operatorname{var}(x)=\sum_{h=1}^{3} \frac{\operatorname{var}_{E h} w_{h}}{N} \\
& \operatorname{var}(x)=\frac{(10082 * 0.35)+(1922 * 0.38)+(326432 * 0.27)}{34} \\
& \operatorname{var}(\bar{x})=2668
\end{aligned}
$$

El error estándar es:

$$
\begin{aligned}
e e_{(x)} & =\sqrt{\operatorname{var}(\bar{x})} \\
e e_{(x)} & =\sqrt{2668} \\
e e_{(x)} & =52
\end{aligned}
$$

El ancho del intervalo de confianza es:

$$
\begin{aligned}
& \bar{x} \pm t_{n-1} e e(x) \\
& =2.6 * 52=133
\end{aligned}
$$

El coeficiente de variación:

$$
\begin{aligned}
& c v=\frac{e e}{\bar{x}} 100 \\
& c v=\frac{51.6}{2461} 100=2 \%
\end{aligned}
$$

